\title{
Estilos de Aprendizagem: proposta de questionário semiautomático de apoio à aprendizagem autorregulada
}

\author{
Antonio Carlos Zambon ${ }^{1}$, Enzo Juniti Fujimoto ${ }^{1}$, Higor Augusto Gomes ${ }^{1}$ \\ aczambon@unicamp.br; e233930@dac.unicamp.br; h146435@dac.unicamp.br \\ ${ }^{1}$ Universidade Estadual de Campinas, R. Paschoal Marmo, 1888 - Jd. Nova Itália, 13484-332, Limeira, SP, \\ Brasil
}

DOI: 10.17013/risti.42.136-151

\begin{abstract}
Resumo: Este trabalho descreve o desenvolvimento de um questionário eletrônico semiautomático, baseado no algoritmo do Processo de Análise de Estilos de Aprendizagem por meio de Mapas Conceituais Estendidos. Sua implementação representará uma alternativa para a análise dinâmica dos estilos de aprendizagem e capacitação de alunos para produzirem sua autoavaliação e a aprendizagem autorregulada. No desenvolvimento do questionário foi utilizado o Google Planilhas para a obtenção e armazenamento dos dados e o CmapTools para sua visualização. Os resultados preliminares, demonstrados no caso conceito, confirmam que o questionário é capaz de revelar e representar adequadamente os Estilos de Aprendizagem. Diante disso, a sequência desta pesquisa, consistirá na aplicação extensiva do questionário, com o objetivo de identificar a acuracidade na avaliação dos Estilos de Aprendizagem e o desenvolvimento de relatório para orientação do aluno em suas estratégias de aprendizagem.
\end{abstract}

Palavras-chave: Estilos de Aprendizagem; Mapas Conceituais Estendidos; Aprendizagem Autorregulada; Autoeficácia; Autodidatismo.

\section{Learning Styles: proposal for a semiautomatic support questionnaire for self-regulated learning}

\begin{abstract}
This work describes the development of a semiautomatic electronic questionnaire, based on the Learning Styles Analysis Process algorithm using Extended Concept Maps. Its implementation will represent an alternative for the dynamic analysis of Learning Styles and the training of students to produce their self-assessment and also self-regulated learning. We use Google Spreadsheets for develop the questionnaire and for the storing data, and CmapTools for visualization. The preliminary results, demonstrated in the concept case, confirm that the questionnaire is able to reveal and adequately represent the Learning Styles. Therefore, it is intended that in the next phase of this research, an wide application of the questionnaire will be carried out, with the objective of identifying the accuracy of the Learning Styles identifying process, and the development of a report to guide the student in his learning strategies.
\end{abstract}


Keywords: Learning Styles; Extended Concept Maps; Self-Regulated Learning; Self-efficacy; Autodidactism.

\section{Introdução}

Embora a aprendizagem norteie a evolução humana, muitas são as dificuldades enfrentadas pelos indivíduos no decorrer desse processo. A aprendizagem não se comporta com o mesmo padrão em todos os indivíduos, pois depende da competência cognitiva individual. Alguns indivíduos manifestam uma capacidade autorreflexiva inata, que estimula a evolução do aprendizado diante de necessidades diferentes, o que os torna muito capazes para tratar da realidade complexa. Entretanto, outras pessoas reagem à complexidade de maneira menos efetiva, aprendendo por meios alternativos, nem sempre tão eficientes.

Entende-se como aprendizagem, a capacidade do indivíduo em incorporar ou renunciar formações e racíocinio às suas competências, com o objetivo de construir ou sustentar sua concepção acerca da realidade, positiva ou negativamente.

Essa capacidade também pode ser descrita a partir de um conjunto peculiar de habilidades, manifestas por meio de ações e emoções, que buscam a mudança de um determinado contexto. Essa característica é definida por Marini \& Boruchovitch, 2014; Schunk, 1991; Zimmerman, 1990, como Aprendizagem Autorregulada. Na Aprendizagem Autorregulada, os indivíduos balizam suas aprendizagens e motivações, para atingir os objetivos pessoais. Dessa maneira, a aprendizagem não se torna unicamente acessória e tampouco estática, mas, ativa e dinâmica, por estar no âmago das realizações sociais do indivíduo.

A maneira como o indivíduo estabelece a relação entre o que o ambiente oferece para aprendizado e o que, sob seu julgamento, é relevante, origina a abordagem dos Estilos de Aprendizagem (EA). Os EA, em termos gerais, representam padrões com os quais os indivíduos direcionam suas preferências para aprenderem. Essas preferências evoluem, conforme a compreensão do ambiente pelo indivíduo e reforçam a composição das estratégias que serão por ele utilizadas (Kolb \& Kolb, 2005).

A psicologia da educação (Gatti, 2010) ratifica a importância do estudo da relação do indivíduo e o contexto no qual ele está inserido, pois é dessas relações que emergem os padrões e competências cognitivas, comportamentais e motivacionais, construtoras de suas habilidades. Nesse aspecto, estabelece que a autogestão dos indivíduos sobre seu desenvolvimento, constrói uma aprendizagem robusta, apoiada no autoconceito, que, por sua vez, consolida a gestão do próprio conhecimento (Wernick, 1959). O autoconceito é, normalmente, assumido como um atributo que o indivíduo utiliza para correlacionar a atitude de aprender a um bom desempenho acadêmico ou adaptação profissional (Jackson et al., 2001). Por sua vez, os EA, constituem indicadores importantes para identificação dessa tendência (Omar et al., 2015).

Usualmente, testes para a identificação de EA são estáticos, ou seja, as perguntas não se modificam. Essa característica constitui uma fragilidade, pois, não permite que as mesmas pessoas sejam submetidas diversas vezes ao mesmo questionário, considerando que as respostas podem ser manipuladas pelos respondentes. Os EA são dinâmicos e 
poderiam revelar as mudanças das preferências dos aprendizes expostos aos processos de ensino, se contassem com questionários interativos, customizáveis e dinâmicos.

Uma proposta que atende à possibilidade de tornar os questionários customizáveis e interativos é o Processo de Análise de EA por meio de Mapas Conceituais Estendidos MCE (Gomes, 2018). Esse processo descreve a utilização dos MCE, como ferramentas capazes de extrair e representar conhecimento, revelando as características do EA de uma pessoa. Seu algoritmo de elicitação do conhecimento, possui uma estratégia de criação de perguntas a partir de declarações do próprio respondente, que o torna interativo e customizável, considerando que não utiliza apenas perguntas previamente definidas. Esse processo, no entanto, não foi automatizado, tendo sido descrito apenas seu algoritmo.

O objetivo deste artigo é descrever o desenvolvimento de um questionário eletrônico semiautomático, baseado no algoritmo do Processo de Análise de EA por meio de MCE, que permitirá realizar a análise dinâmica dos estilos de aprendizagem de indivíduos. A ferramenta representará um recurso importante para identificação da mudança de preferências de aprendizagem das pessoas quando expostas a um processo de ensino (López et al., 2016).

Pressupõe-se que, por meio da revelação do EA e da possibilidade do acompanhamento de sua evolução, os indivíduos poderão desenvolver a aprendizagem autorregulada, estimulando-se ao desenvolvimento da autoeficácia (Boruchovitch, 1999).

Este trabalho está descrito em outras quatro seções, além desta introdução. Na Seção 2, apresenta-se a aprendizagem autorregulada e autoeficácia, que norteiam e contextualizam esta proposta no ambiente da aprendizagem. Na Seção 3, descreve-se o desenvolvimento do questionário semiautomático, objeto deste trabalho. Na Seção 4, apresenta-se um caso de conceito cujo objetivo foi testar as funcionalidades da ferramenta. A Seção 5 apresenta as conclusões finais.

\section{Aprendizagem autorregulada e autoeficácia}

Concebe-se que a aprendizagem autorregulada decorre das vivências anteriores do indivíduo, que se reporta a cenários onde ele obteve resultados satisfatórios. Nessa circunstância, são combinados dados gerais de aprendizagem com elementos disposicionais. Esses elementos representam as relações entre eventos ocorridos em momentos diferentes, presentes em uma construção mental abrangente que, por sua vez, é associada por similaridade, a um evento corrente (Lazzeri \& Oliveira-Castro, 2010). Dessa maneira, a aprendizagem autorregulada emerge das competências construídas baseadas na aprendizagem disposicional e inclui comportamentos, atitudes e valores, que conduzem o indivíduo para o conceito de autoeficácia (Jackson et al., 2001; Lazzeri \& Oliveira-Castro, 2010).

Para acreditar que é capaz de aprender, o indivíduo deve possuir uma carga pregressa de elementos com os quais possa construir seu conceito de autoeficácia (Drysdale \& McBeath, 2018; Trautner \& Schwinger, 2020; Yusuf, 2011). Essa característica é relevante para a análise do aprendizado de adultos, que se utilizam de suas experiências anteriores para 
conduzir seu aprendizado (Martín-García et al., 2019). Essas experiências, por vezes, estão associadas à autoeducação, ou autodidatismo.

Os trabalhos existentes no campo do autodidatismo, se referem, em sua grande maioria, à aprendizagem informal. Essa informalidade, concebe o ambiente de aprendizagem como destituído de critérios previamente estabelecidos por um instrutor e restrito à construção de habilidades e ao conhecimento aplicado (Hager, 2000). Entretanto, em abordagens mais recentes, o autodidatismo não se restringe aos meios construtores de habilidades, mas, configura-se como o instrumento pelo qual o indivíduo constrói um ambiente de conhecimento do qual emerge seu autodesenvolvimento, sua imagem particular de mundo, e, consequentemente, sua autorrealização (Selwyn et al., 2016; Shuklina, 2001).

A aprendizagem não se restringe a uma concepção endógena do indivíduo, mas aos construtos que se originam internamente e se expandem para o ambiente externo, buscando sua significação (López et al., 2020). Essa proposição foi amplamente discutida na Teoria Social Cognitiva, cujos estudos do comportamento apontam que a aprendizagem autorregulada tem suas limitações, que são definidas pelo contexto (Alonso et al., 1995; Bandura, 1989). A teoria enfatiza que, durante o autoconhecimento, o indivíduo desenvolve aptidões consistentes para gerenciar sua manutenção, como a motivação empenhada em determinado tópico, cabendo-lhe "formatar-se" em cada ambiente (Yusuf, 2011). Isso está intrinsecamente relacionado à característica humana de reação às pré-concepções sobre os quadros futuros, que provoca uma sensação de desconhecimento, determinante para o planejamento acerca das próprias satisfações, sinalizando o engajamento mais assertivo (Trautner \& Schwinger, 2020).

Assim, no âmbito da aprendizagem, a característica intencional de tomadas de decisões que sejam mais eficientes, não está circunscrita ao ambiente externo. Interpreta-se que, ao se reconhecer pela autoeficácia, e regular-se dentro do contexto, o indivíduo influenciará suas condutas futuras, escolhendo seu Estilo de Aprendizagem.

\subsection{Estilos de Aprendizagem}

Estilos de Aprendizagem (EA) são táticas empregadas pelas pessoas que buscam mitigar problemas ou aumentar a taxa de sucesso frente às dificuldades de aprender (Meurer et al., 2018). Essas táticas são definidas por uma preferência inconsciente, e são escolhidas por comparação a vivências anteriores.

O Modelo de Aprendizado Experiencial (Cavanagh, Hogan e Ramgopal, 2017; Ka \& Chan, 2012; Widiastuti \& Budiyanto, 2018) define o aprendizado como um processo no qual o conhecimento é construído por meio da experiência. A experiência representa a oportunidade de o indivíduo vivenciar os fenômenos que necessita aprender, pensar sobre eles e projetar ações transformadoras. O modelo descreve que o aprendizado de adultos ocorre por meio de um ciclo que considera as preferências dos indivíduos em interagir com os fenômenos que precisam ser aprendidos. São quatro preferências: i) Experiência Concreta; ii) Conceituação Abstrata; iii) Experimentação Ativa e iv) Observação Reflexiva. 
O ciclo propõe que os indivíduos inconscientemente preferem interagir com o ambiente externo. Isso não significa que não possam modificar tais preferências ao longo do tempo, com a maturidade ou, aprendizado. Essas preferências podem ser representadas em dois eixos perpendiculares (Figura 1).

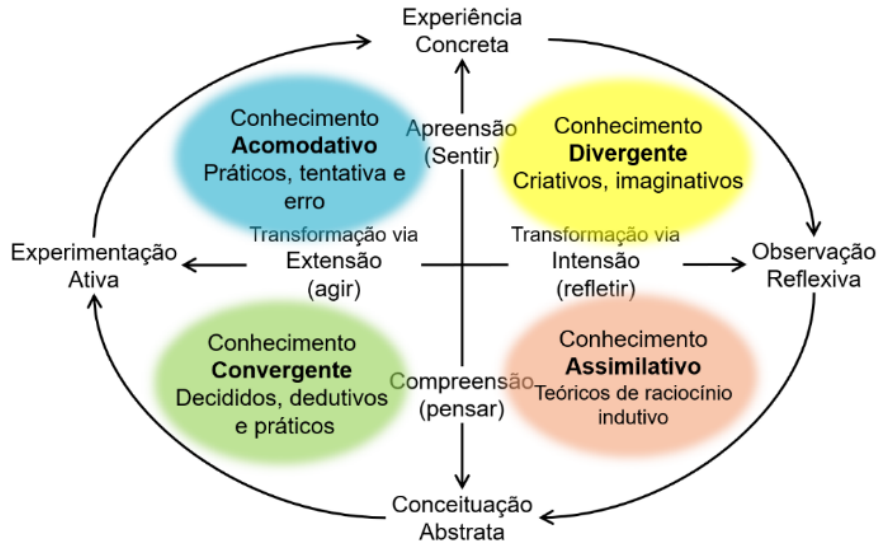

Figura 1 - Ciclo de Aprendizagem e Estilos de Aprendizagem Fonte: adaptado (Nogueira et al., 2012)

Conforme a Figura 1, o eixo vertical representa a Experiência Concreta em uma extremidade e o seu oposto, a Conceituação Abstrata, na outra. Os indivíduos que preferem a Experiência Concreta confiam mais em seus sentidos e buscam aprender pelo contato, pela visualização. Por outro lado, os indivíduos que preferem a Conceituação Abstrata, são capazes de utilizar a reflexão para compreender antes de agir.

Nas extremidades do eixo horizontal se posicionam a Experimentação Ativa e a Observação Reflexiva. Os indivíduos que preferem a Experimentação Ativa para aprender, têm agilidade para a ação modificadora do ambiente, ao passo que os indivíduos que preferem a Conceituação Abstrata, necessitam compreender antes de agir. São mais reflexivos e demandam mais tempo para a ação.

Considerando preferências dispostas nos dois eixos, são definidos quatro estilos de aprendizagem: i) Divergentes, cujos pontos fortes são a criatividade e a imaginação; ii) Assimiladores, que preferem a criação de modelos teóricos, que demandam raciocínio indutivo; iii) Convergentes, que se destacam na solução de problemas, tomada de decisões e aplicação prática de ideias e iv) Acomodadores, que preferem experiências práticas às abordagens teóricas.

O Modelo de Aprendizagem Experiencial (Kolb \& Kolb, 2005) sustenta que a aprendizagem efetiva, se orienta por um movimento cíclico, conduzindo o indivíduo pelos quatro estilos de aprendizagem, e, embora os aprendizes prefiram um estilo em detrimento dos outros, também terão traços dos outros estilos. 


\subsection{Mapas Conceituais Estendidos e a representação de Estilos de Aprendizagem}

Mapas Conceituais estendidos (MCE) são representações gráficas que utilizam regras cognitivas para representação do conhecimento. São estruturados por meio de proposições inter-relacionadas e dispostas em uma matriz que associa o censo de julgamento de um indivíduo sobre si e sobre o ambiente (Galindo-Jaramillo, 2018; Gomes, 2018; Silva, 2018). Os Mapas Conceituais Estendidos (MCE) são representações do conhecimento, e ilustram graficamente as relações existentes entre os conceitos de um domínio do conhecimento (Zambon et al., 2016). Conceitos são palavras ou pequenas frases cujo significado remete a construtos físicos ou abstrações, como sentimentos. Os conceitos são relacionados por meio de frases de ligação (verbos, preposições) inseridos em arcos (arestas) que os unem (Mayer, 2002). Dois conceitos conectados por um arco dão origem a uma proposição, sendo essa a estrutura básica dos MCE.

As proposições recebem dois tipos de sinais: (+) que representam reforço, (-) que representa balanceamento. O reforço $(+)$ descreve uma relação diretamente proporcional entre causa e efeito: quanto mais causa, mais efeito. O peso de balanceamento (-), descreve uma relação inversamente proporcional entre causa e efeito: quanto mais causa, menos efeito (Meadows et al., 2004; Senge, 2006). A Expressão 1 exemplifica uma proposição conforme o MCE.

$$
C_{c} \nabla^{v+} C_{e}
$$

A proposição descrita na Expressão 1, pode ser lida como "Conceito Causa influencia amplificando Conceito Efeito”. O raciocínio de um indivíduo é representado por meio de inúmeras proposições, que são organizadas em uma matriz de atributos.

A matriz é composta por três linhas e três colunas, que descrevem dois contextos: i) as linhas (L), representam a capacidade que um indivíduo julga ter de manipular conceitos, racional ou irracionalmente e obter um resultado previamente imaginado; ii) as colunas (C), representam a hipótese imaginada pelo indivíduo do ambiente se comportar de maneira esperada, em consequência de padrões por ele previamente pensados, em consonância com suas experiências anteriores e suas regras inconscientes. Esses atributos evoluem entre o plano consciente, que na matriz é representado nas intersecções L-C em branco, e o plano inconsciente, representado nas intersecções L-C em cinza.

As proposições possuem seu significado intrínseco, dado pelas palavras e pela estrutura de relacionamento. Entretanto, quando essas proposições são posicionadas na matriz de atributos, recebem o significado desse posicionamento (extrínseco), que associa a relação com as impressões do indivíduo sobre sua capacidade e sobre o ambiente, de acordo com a argumentação (consciente) ou o raciocínio (inconsciente).

O atributo da linha superior da matriz é Controlável (CT), e nela se posicionam conceitos julgados controláveis por regras explícitas. A linha mediana, abrange, além da argumentação (elementos explícitos), também elementos semânticos (tácitos), provenientes do raciocínio. Nessa linha é possível observar o trânsito entre o consciente 
e o inconsciente do indivíduo. Essa condição de transição estabelece que seu nível de controlabilidade é indefinido, por isso, é denominada Penumbra (PN). A linha inferior é composta por elementos semânticos (tácitos) e os conceitos nela posicionados, estão sujeitos a regras que emergem da inconsciência, logo, são Não Controláveis (NC).

$\mathrm{O}$ atributo da coluna mais à direita, é de controlabilidade (CT), representando elementos provenientes de inferência. Esses elementos explicam a dinâmica do ambiente exposto às ações do agente e, segundo seu julgamento, consistem em previsões certas e objetivas. A coluna central, de penumbra (PN), agrupa elementos conscientes e inconscientes, projetando incerteza sobre as regras de mudança do ambiente imaginadas pelo indivíduo. Deixam de ser integralmente inferências, pois possuem elementos do seu raciocínio, da percepção, apoiadas parcialmente na experiência individual, que não é integralmente reconhecida por outros indivíduos. A coluna à esquerda, agrupa elementos não controláveis (NC) pelo indivíduo, que estão posicionados no seu inconsciente. Isso significa que não há explicação lógica dos seus resultados, e nem a origem desses elementos é determinística, pois emergem de regras não declaráveis, subconscientes (Figura 2).

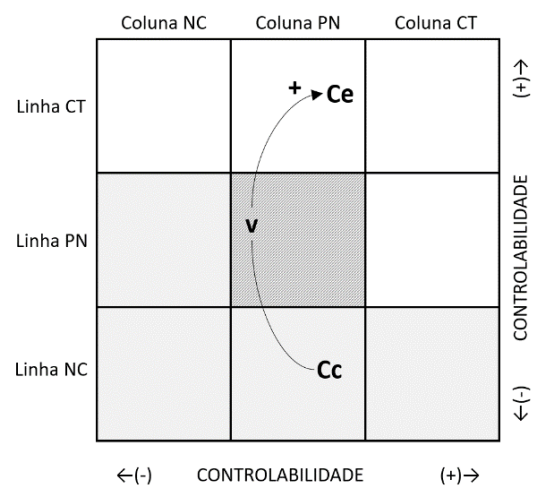

Figura 2 - Mapa Conceitual Estendido Fonte: Adaptado de (Duarte, 2016)

Na exemplificação da Figura 2, é possível identificar a Expressão 1 (Cc $\nabla v+C e) .0$ conceito causa $(\mathrm{Cc})$ se encontra no inconsciente, gerando desestabilização no conceito efeito (Ce). Além disso, o Cc, é tido pelo indivíduo como não controlável (NC) e seu comportamento no ambiente não é claro (PN) para o observador. Embora seja uma verdade, essa consideração influencia a mudança do conceito efeito (Ce) sob condições inesperadas. O Conceito Efeito (Ce), por mais que, retoricamente, se posicione em ambiente controlável, (CT), pelo fato de receber uma carga de desestabilização (+) do conceito causa $(\mathrm{Cc})$, muda seu estado de maneira imprevista. Esse tipo de relacionamento explica, em parte, a frustração pela certeza que não se concretiza.

Essas características de representação permitem, por meio de inserção de atributos específicos na matriz do MCE, identificar estilos de aprendizagem (EA) (Gomes, 2018). 


\section{Desenvolvimento do Formulário semiautomático}

O formulário semiautomático coletará proposições a partir de um questionário interativo, aplicado individualmente e posicionará essas proposições no MCE.

Para o desenvolvimento foi escolhida a plataforma do Google Planilhas. As informações obtidas serão armazenadas em planilha eletrônica e depois exportadas em formato legível para o CmapTools, que será o instrumento de visualização gráfica das proposições coletadas.

A ferramenta para aquisição e representação de Estilos de Aprendizagem é composta por dois processos: i) Processo de Elicitação de Proposições e ii) Processo de Representação no MCE.

\subsection{Processo de elicitação de proposições}

A elicitação de proposições ocorre pela aplicação desenvolvida no Google Planilhas (Google, 2019). A escolha da plataforma se deve à sua flexibilidade, possibilidades de customização, comunicação em tempo real com o banco de dados e capacidade de automatização de processos de extração e tratamento de texto. A interface do formulário eletrônico pode ser observada na Figura 3.

\section{FORMULÁRIO - ESTILOS DE APRENDIZAGEM}

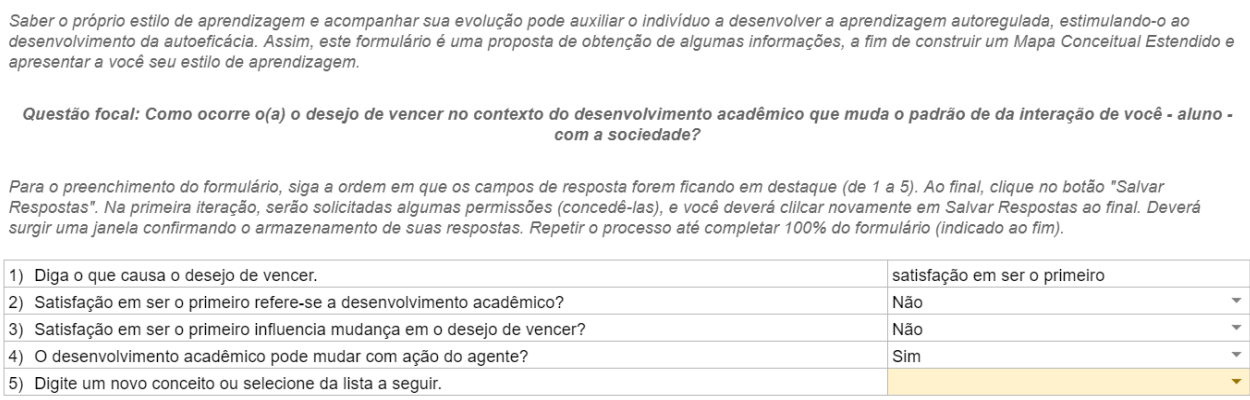

Figura 3 - Formulário de elicitação de proposições

A interação do usuário com o formulário eletrônico (Figura 3), consiste em preencher cinco campos com conceitos que remetem à Questão Focal (Qf), presente no formulário. As perguntas são construídas com conceitos presentes na Qf e na argumentação do respondente. Isso torna o questionário diferente para cada respondente. A cada interação, o usuário adiciona uma proposição $(\mathrm{Cc} \otimes \mathrm{v}+\mathrm{Ce})$, além dos elementos necessários para seu posicionamento na matriz do MCE.

A estrutura do questionário atende às regras de posicionamento de conceitos no MCE, como descrito na Figura 4. 


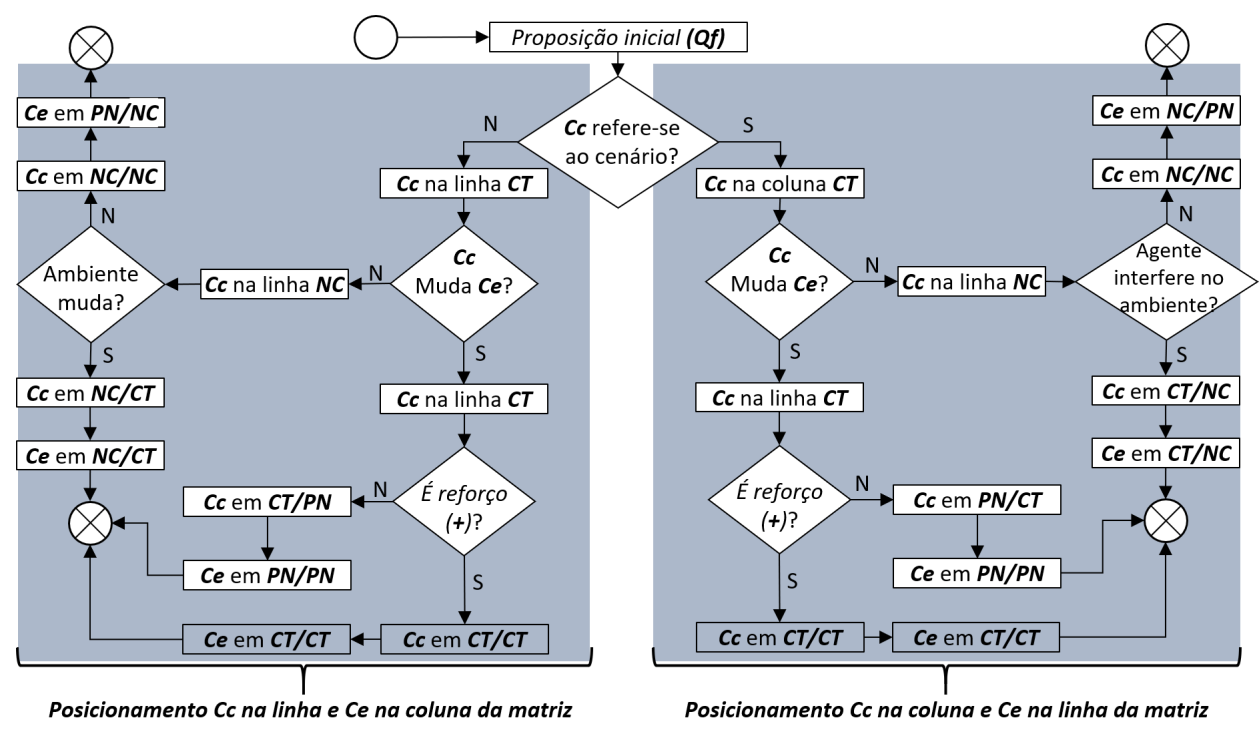

Figura 4 - Algoritmo de posicionamento de conceitos no MCE

Fonte: Adaptado (Gomes, 2018)

É possível identificar na Figura 4, os elementos de decisão, que são percorridos ao longo de cada interação no questionário. Cinco questionamentos básicos são demandados para cobrir as alternativas do questionário:

- Pergunta 1: identifica se Ce, presente na Qf se associa ao Cc definido pelo respondente;

- Pergunta 2: identifica se o Cc, pela ótica do respondente, explica o cenário, ou é circunscrita à capacidade do respondente;

- Pergunta 3: identifica se, em quaisquer das alternativas anteriores, Cc muda o estado do Ce;

- Pergunta 4: identifica se a relação ambiente - agente provoca mudanças em um ou em outro;

- Pergunta 5: identifica se existe outro conceito para adicionar à argumentação do agente, ou, se algum conceito já adicionado pode ser melhor discutido ou associado.

A elicitação cessa após cinco iterações, que representam um número suficiente de proposições para identificação do EA do respondente.

Um fator decisivo para a escolha do Google Planilhas (Google, 2019) como plataforma para a construção do formulário, é a dinâmica das perguntas 2, 3 e 4, que são construídas de maneira interdependente. Por exemplo, a pergunta 3, é construída com elementos sintáticos da resposta à pergunta 2, e a pergunta 4, é construída com elementos sintáticos da resposta à pergunta 3 . 
Ao final da elicitação, as respostas são armazenadas em uma tabela inicial que preserva a estrutura da proposição, além da localização do Conceito Causa (Cc), do Conceito Efeito (Ce) e do Verbo (v), para que possam ser posicionados na matriz de atributos do MCE, conforme proposto pelo algoritmo de posicionamento. A Tabela 1 descreve o exemplo de duas proposições e os posicionamentos X-Y dos conceitos na matriz de atributos.

\begin{tabular}{lllllllll}
\hline $\begin{array}{l}\text { Conceito Causa } \\
\text { (Ce) }\end{array}$ & Verbo (v) & $\begin{array}{l}\text { Conceito Efeito } \\
\text { (Ce) }\end{array}$ & Cc & v & & Ce \\
\hline \multirow{2}{*}{ Stress nas relações } & Influencia & Produtividade & 1250 & 478 & 221 & 413 & 105 & 963 \\
\hline Má gestão & Influencia & Motivação & 520 & 665 & 150 & 1075 & 963 & 783 \\
\hline
\end{tabular}

Tabela 1 - Base de proposições e posicionamento dos conceitos

Da esquerda para a direita, a Tabela 1 apresenta conceitos causa (Cc), os verbos (v) e os conceitos efeito (Ce). As colunas posteriores contém as informações de posicionamento (linha e coluna) dos três elementos anteriores no MCE.

Os dados finais são exportados em formato tsv, ou Valores Separados por Tabulação, e na sequência são convertidos para csv, ou Valores Separados por Vírgula. Esse processo é realizado com a finalidade de preservar a codificação UTF-8, que possibilita o uso de acentos gráficos.

\subsection{Processo de representação no MCE}

A base de proposições e seus posicionamentos são exportados para o CmapTools (IHMC, 2020), onde serão representados no formato da matriz de atributos do MCE.

A partir de uma análise do algoritmo de posicionamento, é possível identificar oito hipóteses de caminhos para posicionamento dos conceitos no MCE. O caminho percorrido pelas respostas, recebe uma etiqueta, ou carimbo, definido pela sequência de Sim e Não. Os carimbos, portanto, são caminhos gerados pelo respondente, sob ação do algoritmo. No caso específico de um carimbo SimSimSim, obtém-se o posicionamento dos conceitos Causa e Efeito, tanto para linha quanto para a coluna (Tabela 2).

\begin{tabular}{lllll}
\hline Carimbo & Linha CC & Coluna CC & Linha CE & Coluna CE \\
\hline SimSimSim & Controlável & Controlável & Controlável & Controlável \\
\hline SimSimNão & Penumbra & Controlável & Penumbra & Penumbra \\
\hline SimNãoSim & Controlável & Não Controlável & Controlável & Não Controlável \\
\hline SimNãoNão & Não Controlável & Não Controlável & Não Controlável & Penumbra \\
\hline NãoSimSim & Controlável & Controlável & Controlável & Controlável \\
\hline
\end{tabular}




\begin{tabular}{lllll}
\hline Carimbo & Linha CC & Coluna CC & Linha CE & Coluna CE \\
\hline NãoSimNão & Controlável & Penumbra & Penumbra & Penumbra \\
\hline NãoNãoSim & Não Controlável & Controlável & Não Controlável & Controlável \\
\hline NãoNãoNão & Não Controlável & Não Controlável & Penumbra & Não Controlável \\
\hline
\end{tabular}

Tabela 2 - Possibilidades de posicionamento dos conceitos

Na Tabela 2, é possível identificar oito carimbos, posicionados em linha. Cada carimbo, define um caminho para cada decisão do fluxograma.

Para cada carimbo, estabelecem-se os limites de posicionamento na matriz do MCE, que está associado à relação linha-coluna da matriz, como demonstrado na Figura 7.

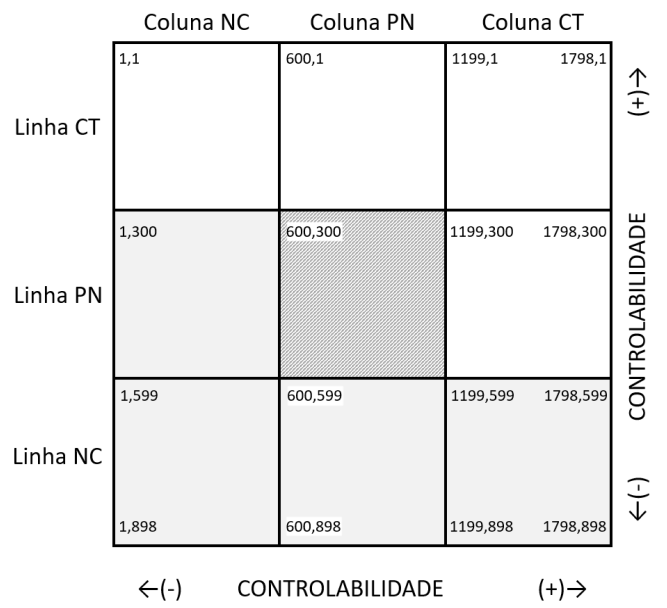

Figura 7 - Limites de posicionamento dos conceitos no Mapa Conceitual Estendido

Na Figura 7, os valores nas legendas de cada quadrante são as coordenadas limites no MCE (X-Y). Por exemplo, um conceito inserido nas coordenadas entre $(1199,1)$ e $(1798,1)$, será posicionado como Controlável.

Dessa maneira, os conceitos e os relacionamentos são posicionados na matriz de atributos do MCE. A partir desse posicionamento, é possível identificar os Estilos de Aprendizagem dos respondentes.

\section{Resultados obtidos}

Após seu desenvolvimento, o questionário foi submetido a testes para avaliação de sua capacidade de posicionar automaticamente conceitos na matriz do MCE, como proposto 
pelo algoritmo de posicionamento de conceitos (Figura 4). Na Figura 9, observa-se uma matriz construída em um dos testes realizados, onde é possível identificar os conceitos e seus relacionamentos nos eixos "Concreto-Abstrato" e "Ativo-Reflexivo", como preconizado pelo Processo de Análise de EA por meio de MCE (Gomes, 2018).

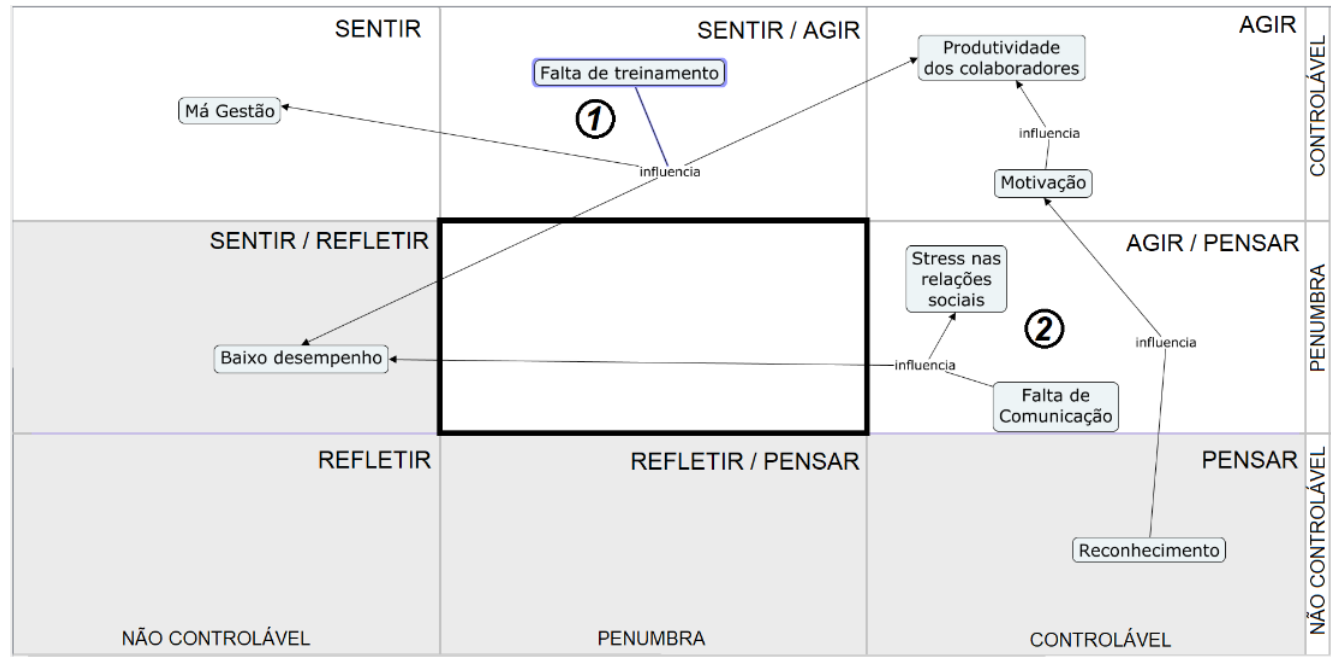

Figura 9 - Matriz do MCE com os atributos do Ciclo de Aprendizagem

Na Figura 9, as preferências Agir e Sentir, se situam em Experiência Ativa e Concreta, e se posicionam na área do Consciente (branco) do MCE. As preferências Refletir e Pensar referem-se à Conceituação Abstrata e Observação Reflexiva, e por essa razão, se encontram no âmbito do Inconsciente (cinza). As relações L-C intermediárias, são definidas como áreas de transição entre os eixos.

Os MCE são construídos por várias proposições e, para definir o EA a partir desses conjuntos, é utilizada a estratégia de Análise de Hubs (Galindo-Jaramillo, 2018; Gomes, 2018).

Uma relação no padrão hub ocorre quando um conceito é causa de vários conceitos. Nessa hipótese, o hub é chamado de difusor (HD). Em outra circunstância, quando vários conceitos são causa de um único, o hub é denominado concentrador (HC).

Na análise de EA são utilizados apenas os HD. Na Figura 9 estão representados o HD1 e HD2. No HD1, o conceito "Falta de treinamento" influencia outros três conceitos: "Má gestão", "Baixo desempenho" e "Produtividade dos colaboradores". O HD1 é predominante, comparativamente ao HD2, que influencia dois conceitos. O HD1 se encontra na intersecção L-C "Sentir/Agir", que denota o EA predominante "Acomodativo" (Tabela 2). 


\begin{tabular}{lllllc}
\hline HD & Cc & RELAÇÕES & LOCALIZAÇÃo & EA - Kolb & \% \\
\hline 1 & $\begin{array}{l}\text { Falta de } \\
\text { Treinamento }\end{array}$ & 3 & Sentir/Agir & Acomodativo & $60 \%$ \\
\hline 2 & $\begin{array}{l}\text { Falta de } \\
\text { Comunicação }\end{array}$ & 2 & Agir/Pensar & Convergente & $40 \%$ \\
\hline & Total & 5 & & & $100 \%$ \\
\hline
\end{tabular}

Tabela 2 - Definição de Estilos de Aprendizagem pela análise da formação de Hubs no MCE

A Tabela 2 contém a análise dos dois HD existentes no MCE da Figura 9. Observa-se que o HD1 é predominante (60\%), porém, o HD2 é significativo (40\%), não devendo ser descartado na análise do indivíduo.

\section{Conclusões}

O objetivo deste trabalho foi apresentar o processo de desenvolvimento de um formulário eletrônico semiautomático para execução do algoritmo do Processo de Análise de EA por meio de MCE (Gomes, 2018).

O formulário interativo e dinâmico, baseou-se em Google Planilhas (Google, 2019) para a elicitação dos Estilos de Aprendizagem - EA. A visualização dos EA é possibilitada pela exportação de dados em texto para o CmapTools (IHMC, 2020), que converte o arquivo no formato de Mapa Conceitual Estendido (MCE).

Os EA são revelados pela matriz de atributos do MCE e seu formulário customizável permite a introdução de perguntas diferentes a cada elicitação.

Este trabalho analisou, por meio de um caso de conceito, a eficiência do formulário eletrônico em executar o algoritmo do processo estudado, qualificando-o como ferramenta capaz de identificar EA e suas mudanças, ao longo do tempo.

Apoiados por esta ferramenta, indivíduos poderão aprofundar o entendimento sobre si mesmos, frente às dificuldades de aprenderem. Dessa maneira, poderão incrementar a aprendizagem autorregulada e estimularem-se ao desenvolvimento da autoeficácia.

Em termos tecnológicos, houve uma contribuição efetiva, com a criação de um questionário automaticamente conversível em um modelo gráfico de análise. Essa característica abre uma nova frente para a construção de métodos autogerenciáveis, que favoreçam a autoeficácia. Soma-se a isso, a característica dinâmica e customizável dos formulários, que permitem sua utilização incontáveis vezes com os indivíduos e também instanciá-los para vários cenários.

Trabalhos futuros abrangerão a aplicação extensiva do questionário, em diferentes ambientes, como o acadêmico e empresarial, com o objetivo de aferir a acuracidade 
na avaliação dos EA. Essas pesquisas abrangerão ainda, o desenvolvimento de um formulário que possibilite aos alunos, a compreensão e utilização adequada e efetiva da informação para o desenvolvimento de estratégias de aprendizagem. Essas condições são essenciais para o uso efetivo da ferramenta.

\section{Agradecimentos}

O presente trabalho foi realizado com o apoio da Coordenação de Aperfeiçoamento de Pessoal de Nível Superior - Brasil (CAPES) - Código de Financiamento oo1 e do Conselho Nacional de Desenvolvimento Científico (CNPq) - Chamada MCTIC/CNPq No 28/2018 - Universal/Faixa B; e também do SAE-CNPq-Unicamp.

\section{Referências}

Alonso, C., Gallego, D., \& Honey, P. (1995). Los estilos de aprendizaje. Procedimientos de diagnóstico y mejora. In Ediciones Mensajero (8th ed., Vol. 1). Ediciones Mensajero.

Bandura, A. (1989). Human Agency in Social Cognitive Theory. American Psychology, 44 (9), 1175-1184. https://doi.org/10.1037/0003-066X.44.9.1175

Boruchovitch, E. (1999). Estratégias de aprendizagem e desempenho escolar: considerações para a prática educacional. Psicologia: Reflexão e Crítica, 12(2), 361-376. https://doi.org/10.1590/s0102-79721999000200008

Drysdale, M. T. B., \& McBeath, M. (2018). Motivation, self-efficacy and learning strategies of university students participating in work-integrated learning. Journal of Education and Work, 31(5-6), 478-488. https://doi.org/https://doi.org/10.108 $\mathrm{o} / 13639080.2018 .1533240$

Duarte, G. A. (2016). Visualização de Mapas Conceituais Estendidos utilizando grafos orientados a força e restrições de posicionamento de vértices [Dissertação de Mestrado em Tecnologia, Faculdade de Tecnologia, Universidade Estadual de Campinas]. http://repositorio.unicamp.br/handle/REPOSIP/331502

Galindo-Jaramillo, J. F. (2018). Identificação da percepção de valor público por meio de ferramentas de gestão do conhecimento. [Dissertação de Mestrado em Tecnologia, Faculdade de Tecnologia, Universidade Estadual de Campinas]. http://repositorio. unicamp.br/jspui/handle/REPOSIP/334274

Gatti, B. A. (2010). Psicologia da educação: conceitos, sentidos e contribuições. Psicologia Da Educação, 31, 7-22. http://pepsic.bvsalud.org/scielo.php?script=sci_ arttext\&pid=S1414-69752010000200002\&lng=pt\&nrm=iso\&tlng=pt

Gomes, F. D. (2018). Ferramentas de Gestão do Conhecimento e Estilos de Aprendizagem para apoio às estratégias pedagógicas no ensino superior. [Doutorado em Tecnologia, Faculdade de Tecnologia, Universidade Estadual de Campinas]. http://repositorio. unicamp.br/jspui/handle/REPOSIP/333644

Google, (2019). Planilha Google (08/2019; p. Software). Google LCC. https://docs. google.com/spreadsheets/ 
Hager, P. (2000). Know-how and workplace practical judgement. Journal of Philosophy of Education, 34(2), 281-296. https://doi.org/10.1111/1467-9752.00173

IHMC, I. for H. and M. C. (2020). CmapTools (p. Software). IHMC. http://cmap.ihmc.us Jackson, S. A., Thomas, P. R., Marsh, H. W., \& Smethurst, C. J. (2001). Relationships betweenFlow, Self-Concept,PsychologicalSkills, andPerformance.JournalofApplied Sport Psychology, 13(1), 129-153. https://doi.org/10.1080/104132001753149865

Ka, C., \& Chan, Y. (2012). Exploring an experiential learning project through Kolb 's Learning Theory using a qualitative research method. 3797. https://doi.org/10.108 o/03043797.2012.706596

Kolb, A. Y., \& Kolb, D. A. (2005). Learning styles and learning spaces: Enhancing experiential learning in higher education. Academy of Management Learning and Education, 4(2), 193-212. https://doi.org/10.5465/AMLE.2005.17268566

Lazzeri, F., \& Oliveira-Castro, J. M. (2010). Termos psicológicos disposicionais e análise do comportamento. Princípios: Revista de Filosofia (UFRN), 17(28), 155-183.

López, C., Janet, M., Castillo, L., Maldonado, P., Alicia, A., Casados, C., \& Romo, A. (2020). Estrategias para potenciar el aprendizaje y el rendimiento académico en estudiantes universitarios. Revista Venezolana de Gerencia, 25(90), 579-594.

López, M. B., Montes, A. J. H., Ramírez, R. V., Hernández, G. A., Cabada, R. Z., \& Estrada, M. L. B. (2016). EmoRemSys: Sistema de recomendación de recursos educativos basado en detección de emociones. Revista Ibérica de Sistemas e Tecnologias de Informação, 1(17), 80-95. https://doi.org/10.17013/risti.17.80-95

Marini, J., \& Boruchovitch, E. (2014). Estratégias de aprendizagem de alunos brasileiros do ensino superior: Considerações sobre adaptação, sucesso acadêmico e aprendizagem autorregulada. Revista Eletrônica de Psicologia, Educação e Saúde, 1, 102-126.

Martín-García, A. V., Sánchez-Gómez, M. C., \& Pérez, B. G. (2019). Ejemplificación de metodología mixta para el análisis del uso de entornos blended learning en docentes universitarios. Revista Ibérica de Sistemas e Tecnologias de Informação, 1(33), 16-31. https://doi.org/10.17013/risti.33. 16-31

Mayer, R. E. (2002). Rote Versus Meaningful Learning. Theory Into Practice, 41(4), 226-232. https://doi.org/10.1207/s15430421tip4104_4

Meadows, D., Randers, J., \& Meadows, D. (2004). The Limits to Growth: The 30-Year Update (2 ed.). Chelsea Green Publishing Company.

Meurer, A. M., Pedersini, D. R., Antonelli, R. A., \& Voese, S. B. (2018). Estilos de Aprendizagem e Rendimento Acadêmico na Universidade. REICE. Revista Iberoamericana Sobre Calidad, Eficacia y Cambio En Educación, 16(4), 23-43. https://doi.org/10.15366/reice2018.16.4.002

Nogueira, D. R., Espejo, M. M. dos S. B., Reis, L. G. dos, \& Voese, S. B. (2012). Estilos de aprendizagem e desempenho em educação à distância: um estudo empírico com alunos das disciplinas de contabilidade geral e gerencial. Revista De Educação E Pesquisa Em Contabilidade (REPeC), 6(1). https://doi.org/10.17524/repec.v6i1.181 
Omar, N., Mohamad, M. M., \& Paimin, A. N. (2015). Dimension of Learning Styles and Students' Academic Achievement. Procedia - Social and Behavioral Sciences, 204, 172-182. https://doi.org/10.1016/j.sbspro.2015.08.130

Schunk, D. H. (1991). Self-Efficacy and Academic Motivation. Educational Psychologist, 26(1), 207-231. https://doi.org/10.12681/eadd/1834

Selwyn, N., Gorard, S., \& Furlong, J. (2016). Studies in the Education of Adults Adults' use of computers and the Internet for self-education. Studies in the Education of Adults, 38(2), 141-159. https://doi.org/https://doi.org/10.1080/02660830.2006 .11661530

Senge, P. M. (2006). The Fifth Discipline: The Art and Practices of The Learning Organization (1a edição). In Broadway Business.

Shuklina, E. A. (2001). Technologies of Self-Education. Russian Education \\& Society, 43(4), 57-78. https://doi.org/10.2753/RES1060-9393430457

Silva, W. J. da. (2018). Processo de identificação de padrões em modelos mentais com foco no risco de evasão escolar. [Dissertação de Mestrado em Tecnologia, Faculdade de Tecnologia, Universidade Estadual de Campinas]. http://repositorio.unicamp. br/jspui/handle/REPOSIP/335151.

Trautner, M., \& Schwinger, M. (2020). Integrating the concepts self-efficacy and motivation regulation: How do self-efficacy beliefs for motivation regulation influence self-regulatory success? Learning and Individual Differences, 80(101890), 1-13. https://doi.org/10.1016/j.lindif.2020.101890

Wernick, W. (1959). The Self-Concept. Journal of Teacher Education. https://doi. org/10.1177/002248715901000126

Widiastuti, I., \& Budiyanto, C. W. (2018). Applying an experiential learning cycle with the aid of finite element analysis in engineering education. Journal of Turkish Science Education, 15(Special Issue), 97-103. https://doi.org/10.12973/tused.10261a

Yusuf, M. (2011). The impact of self-efficacy, achievement motivation, and selfregulated learning strategies on students' academic achievement. Procedia Social and Behavioral Sciences, 15(1), 2623-2626. https://doi.org/10.1016/j. sbspro.2011.04.158

Zambon, A. C., Baioco, G. B., Chiste, C., \& Vasques, D. G. (2016). Uma aplicação prática de gestão do conhecimento e simulação na resolução de problemas complexos empresariais. Revista Produção Online, 16(2), 408. https://doi.org/10.14488/16761901.v16i2.1799

Zimmerman, B. J. (1990). Self-Regulated Learning and Academic Achievement: An Overview. Educational Psychologist, 25(1), 3-17. https://doi.org/http://dx.doi. org/10.1207/s15326985ep2501_2 